\title{
Erratum to: The Reason for Incommensurability of Three-Phase Theory with Classical Traffic Flow Theories
}

\author{
Boris S. Kerner
}

\section{Erratum to:}

Chapter 8 in: B.S. Kerner, Breakdown in Traffic Networks, DOI 10.1007/978-3-662-54473-0_8

Unfortunately, there was an error in Fig. 8.28(b) in the original publication of the chapter. The original chapter has been corrected. The correct version of the Figure is shown below. 

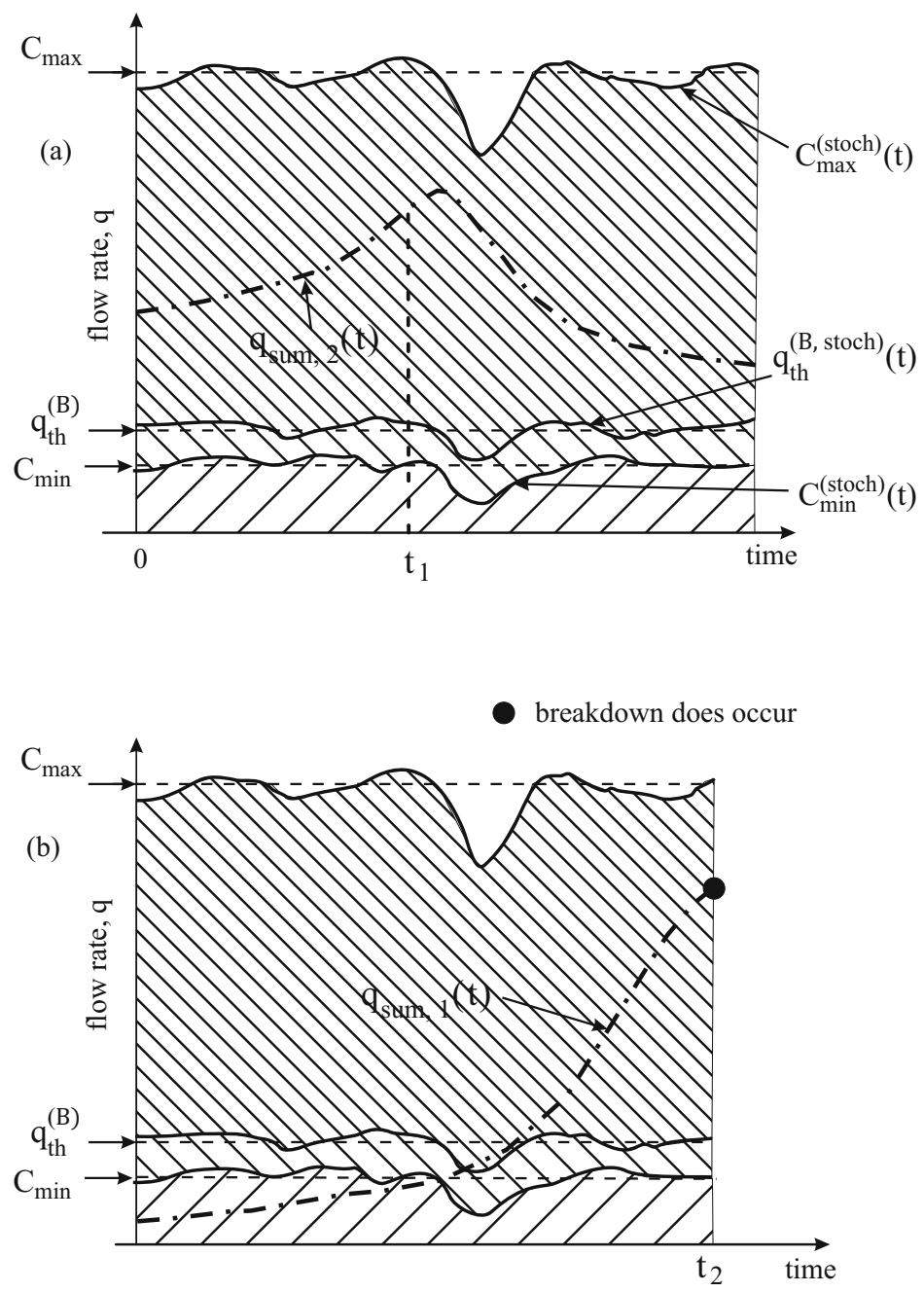

Fig. 8.28 Qualitative explanation of traffic breakdown with the use of the infinite number of capacities of free flow at a highway bottleneck of the three-phase theory. Hypothetical timefunctions $C_{\max }^{\text {(stoch) }}(t), C_{\min }^{\text {(stoch) }}(t)$, and $q_{\mathrm{th}}^{\text {(B, stoch) }}(t)$ are adapted from Fig. 5.14. Hypothetical time functions of the flow rates $q_{\mathrm{sum}, 2}(t)$ in (a) and $q_{\mathrm{sum}, 1}(t)$ in (b) as well as time instant $t_{1}$ in (a) are, respectively, the same as those in Fig. 4.14. Adapted from [67-69] 